14

\title{
Механизмы перехода от ритмической активности к пачечной в модели ноцицептивного нейрона
}

\author{
(C) O.E. Дик \\ Институт физиологии им. И.П. Павлова РАН, \\ 199034 Санкт-Петербург, Россия \\ e-mail: dickviola@gmail.com
}

Поступило в Редакцию 14 марта 2019 г.

В окончательной редакции 4 мая 2019 г.

Принято к публикации 3 октября 2019 г.

Исследованы механизмы перехода от ритмической активности к пачечной в модели мембраны ноцицептивного нейрона при изменении величины внешнего стимулирующего тока. Обнаружено, что наличие седлоузловой бифуркации предельного цикла в структуре бифуркационной диаграммы быстрой подсистемы и бифуркации тора в структуре бифуркационной диаграммы полной системы приводят к возникновению особых решений типа torus canards при этих переходах. Это подтверждает предположение о том, что решения типа torus canards представляют собой обязательную особенность для переходов между ритмическими и пачечными разрядами.

Ключевые слова: бифуркационный анализ, пачечные разряды, бифуркация Андронова-Хопфа, решения типа „утки“, модель ноцицептивного нейрона.

DOI: $10.21883 / J T F .2020 .03 .48939 .93-19$

\section{Введение}

Известно, что нейроны проявляют разнообразные типы поведения, включая ритмическую активность и пачечные разряды различной формы, амплитуды и частоты. Мембрана ноцицептивного нейрона дорсального ганглия млекопитающих также может генерировать пачечные разряды при определенных условиях. Такая эктопическая пачечная активность, не свойственная этим нейронам при нормальных условиях, сигнализирует, как правило, о восприятии болевого повреждающего воздействия $[1,2]$. Поэтому понимание механизмов возникновения этой активности связано с пониманием механизма возникновения нейропатической боли.

Ритмическая и пачечная активность, возникающая в ноцицептивных нейронах дорсальных ганглиев млекопитающих, генерируется в основном $\mathrm{Na}_{V} 1.1, \mathrm{Na}_{V} 1.7$ и $\mathrm{Na}_{V} 1.8$ каналами и каналами задержанного калиевого тока [3-6]. В связи с тем что в ряде экспериментальных работ было показано, что повреждение аксона нейронов дорсальных ганглиев может приводить к снижению экспрессии мРНК калиевых каналов [7] и соответственно к снижению доли калиевых токов различной кинетики [8], в предыдущей работе мы исключили влияние каналов задержанного калиевого тока на возникновение пачечной активности и доказали, что простейшая модель мембраны ноцицептивного нейрона, учитывающая только натриевые токи разной кинетики и токи через каналы утечки, может генерировать и ритмическую, и пачечную активности [9]. Было установлено, что при увеличении стимулирующего тока потеря устойчивости стационарного состояния происходит через субкритическую бифуркацию Андронова-Хопфа, в окрестности которой наблюдаются синглетные колебания с чередованием импульса большой амплитуды с двумя подпороговыми колебаниями малой амплитуды. Увеличение стимула приводит к бифуркации удвоения периода и возникновению пачечных разрядов в виде дуплетов, в которых происходит чередование двух импульсов большой амплитуды с одним подпороговым колебанием. Дальнейшее возрастание стимулирующего тока обеспечивает увеличение числа разрядов в пачке, затем режим пачечных колебаний сменяется на режим ритмических разрядов. Следующее повышение стимула сопровождается потерей устойчивости периодических колебаний через бифуркацию тора и переходом к амплитудно-модулированным колебаниям (пульсирующим волнообразным паттернам с попеременно возрастающей и убывающей амплитудой импульсов), а от них снова к пачечной активности с большим числом импульсов в пачке и длинными интервалами между пачками [9]. При анализе бифуркационных диаграмм быстрой подсистемы было определено, что механизм возникновения пачечной активности в условиях блокирования калиевого тока связан с прохождением траектории системы через бифуркацию Андронова-Хопфа и седло-узловую бифуркацию предельного цикла [9]. В работе [10] было показано, что подобные изменения режимов импульсной активности мембраны ноцицептивного нейрона характерны для относительно широкого диапазона изменения значений плотности медленных натриевых каналов.

Модели нейронов, которые имеют в структуре бифуркационной диаграммы быстрой подсистемы субкритическую бифуркацию Андронова-Хопфа для стационарного состояния и седлоузловую бифуркацию предельного цикла, а также в которых потеря устойчивости пери- 
одических колебаний при изменении бифуркационного параметра происходит в точке бифуркации тора, могут иметь особые решения типа „утки“, которые в иностранной литературе называются torus canards [11]. Решения типа torus canards представляют собой орбиты, проводящие длительное время вблизи притягивающих (устойчивых) и отталкивающих (неустойчивых) периодических орбит [12] и важны для понимания механизма перехода между ритмическими и пачечными разрядами [11-14].

Цель настоящей работы - доказать, что механизмы перехода от ритмической активности к пачечной в модели ноцицептивного нейрона включают решения типа ,Утки“.

\section{1. Модель ноцицептивного нейрона}

Как показано в работе [9], простейшая модель мембраны ноцицептивного нейрона дорсальных ганглиев крыс может включать ток утечки $\left(I_{L}\right)$ и три натриевых тока: быстрый $\left(I_{N a f}\right)$, быстро активирующийся и инактивирующийся ток, промежуточный $\left(I_{N a I}\right)$, быстро активирующийся и медленнее инактивирующийся ток, и медленный $\left(I_{N a S}\right)$, значительно медленнее инактивирующийся ток, генерируемые $\mathrm{Na}_{V} 1.1, \mathrm{Na}_{V} 1.7$ и $\mathrm{Na}_{V} 1.8$ каналами соответственно [15]:

$$
\begin{aligned}
\frac{d E}{d t}= & \left(I-I_{N a f}(h, E)\right. \\
& \left.-I_{N a l}(b, E)-I_{L}(E)-I_{N a s}(s, r, E)\right) / c_{m}, \\
\frac{d x}{d t}= & \left(x_{\infty}(E)-x\right) / \tau_{x}(E), \quad x=h, b, s, r,
\end{aligned}
$$

где $E$ - величина мембранного потенциала, $I$ - стимулирующий ток,

$$
\begin{gathered}
I_{N a f}=g_{N a} m_{\infty}^{3} h\left(E-E_{N a}\right), \\
I_{N a I}(b, E)=g_{N a I} m_{N a I} m_{I \infty}(E) b\left(E-E_{N a}\right), \\
I_{N a S}(s, r, E)=g_{N a S} s^{3} r\left(E-E_{N a}\right),
\end{gathered}
$$

где $c_{m}=1 \mu \mathrm{F} / \mathrm{cm}^{2}, g_{N a}=40 \mathrm{mS} / \mathrm{cm}^{2}, g_{N a I}=27 \mathrm{mS} / \mathrm{cm}^{2}$, $g_{L}=1.4 \mathrm{mS} / \mathrm{cm}^{2}, g_{N a S}=5.7 \mathrm{mS} / \mathrm{cm}^{2}$ - емкость мембраны, проводимости каналов быстрого и промежуточного натриевого токов, каналов утечки и каналов медленного натриевого тока; $E_{N a}=62 \mathrm{mV}, E_{L}=-77 \mathrm{mV}$ - равновесные потенциалы для $\mathrm{Na}^{+}$и ионов утечки. Потенциалзависимые характеристики воротных процессов каналов имеют вид

$$
\begin{gathered}
m_{\infty}(E)=1 /(1+\exp (-(34.1+E) / 9.1)), \\
h_{\infty}(E)=1 /(1+\exp ((56.4+E) / 7.2)), \\
m_{l \infty}(E)=1 /(1+\exp (-(25.3+E) / 9.1)), \\
b_{\infty}(E)=1 /(1+\exp ((72.5+E) / 8)), \\
s_{l \infty}(E)=1 /(1+\exp (-(E+22) / 16)),
\end{gathered}
$$

$$
\begin{gathered}
r_{\infty}(E)=1 /(1+\exp ((E+34) / 11)), \\
\tau_{h}=10.24+1.63 \exp \left(-0.5((E+61.9) / 15.3)^{2}\right), \\
\tau_{b}=0.22 \exp (-0.07 E), \\
\tau_{s}(E)=2+25 \exp \left(-((E+50) / 65)^{2}\right), \\
\tau_{r}(E)=50+250 \exp \left(-((E+21) / 31)^{2}\right) .
\end{gathered}
$$

Численные решения системы (1) находились методом Рунге-Кутты четвертого порядка, модифицированным алгоритмом Гира с выбором переменного шага интегрирования. Механизмы перехода колебаний модели от ритмических к пачечным были определены методом бифуркационного анализа [16]. Для нахождения значений параметров модели, при которых происходят бифуркации предельных циклов, применялось разложение исходной системы на медленную и быструю подсистемы и анализ прохождения траектории периодического решения быстрой подсистемы через бифуркационные значения параметров медленной подсистемы [17] с помощью пакета программ MATCONT [18].

В связи с тем что медленный натриевый ток имеет постоянную времени $\left(\tau_{r}\right)$, значительно превышающую постоянные времени для других токов $\left(\tau_{r} \leq 300 \mathrm{~ms}\right.$; $\left.\tau_{h} \leq 2 \mathrm{~ms} ; \tau_{b} \leq 20 \mathrm{~ms} ; \tau_{s} \leq 25 \mathrm{~ms}\right)$, в качестве быстрой подсистемы рассматривалась система

$$
\begin{gathered}
\frac{d E}{d t}=\left(I-I_{N a f}(h, E)-I_{N a I}(b, E)\right. \\
\left.-I_{L}(E)-I_{N a s}(s, r, E)\right) / c_{m}, \\
\frac{d x}{d t}\left(x_{\infty}(E)-x\right) / \tau_{x}(E), \quad x=h, b, s,
\end{gathered}
$$

в которой переменная инактивации медленного натриевого тока $r$ является медленно меняющимся бифуркационным параметром. Медленная подсистема определялась уравнениями

$$
\begin{gathered}
I-I_{N a f}(h, E)-I_{N a I}(b, E)-I_{L}(E)-I_{N a s}(s, r, E)=0, \\
\frac{d r}{d t}=\left(r_{\infty}(E)-r\right) / \tau_{r}(E) .
\end{gathered}
$$

Соответственно построение однопараметрических бифуркационных диаграмм быстрой подсистемы (2) на плоскости $(r, E)$ позволило определить механизмы смены режимов импульсной активности в модели ноцицептивного нейрона.

\section{2. Результаты}

\section{1. Динамика полной системы: двухпараметрический бифуркационный анализ}

Двухпараметрическая бифуркационная диаграмма на плоскости $\left(I, g_{\mathrm{NaS}}\right)$ показана на рис. 1 . Область ритмических колебаний ограничена двумя кривыми, соответствующими бифуркации Андронова-Хопфа (обозначенными 

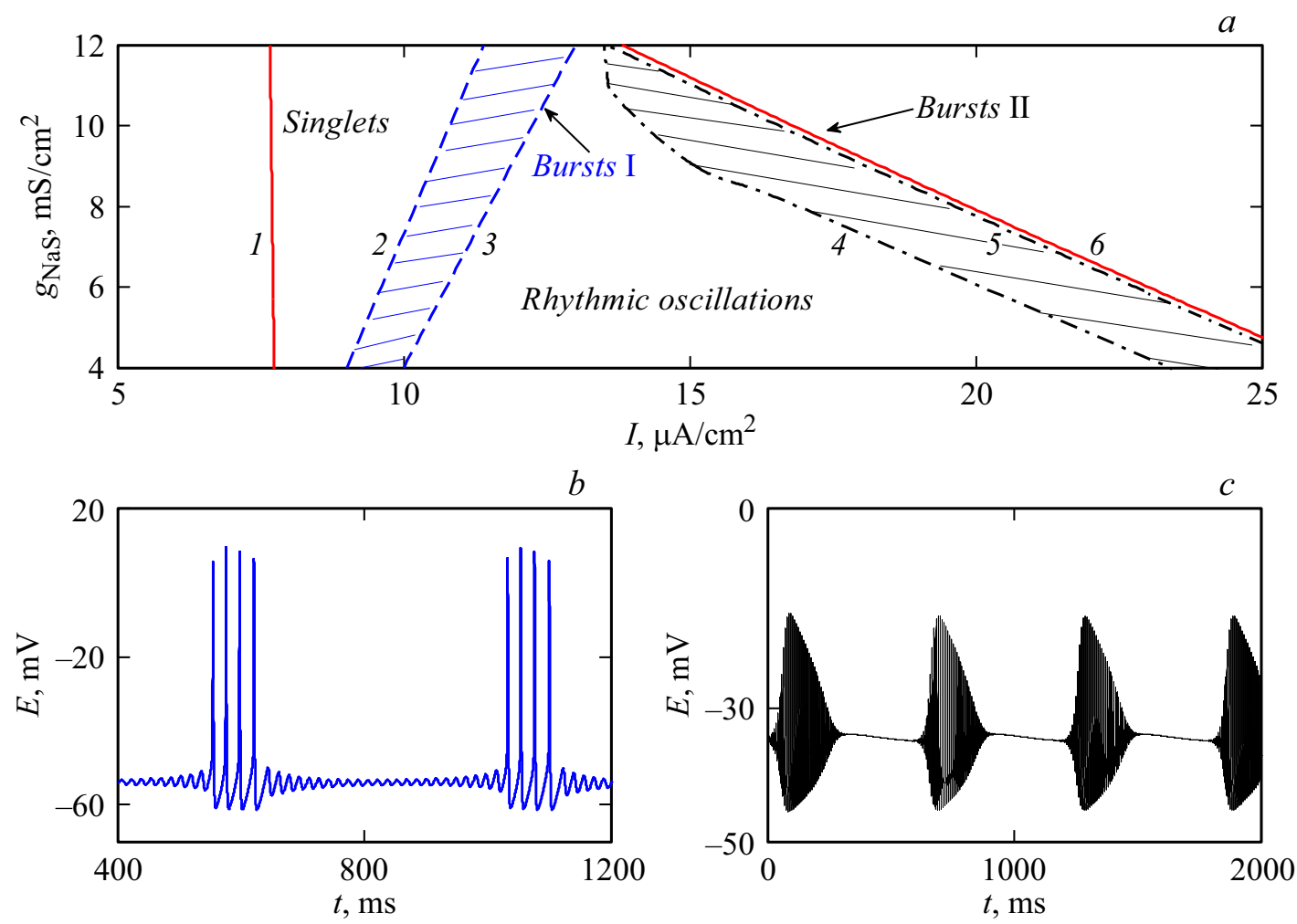

Рис. 1. Бифуркационные кривые системы (1) на плоскости $\left(I, g_{N a S}\right)$ и примеры пачечных разрядов. Область пачечных разрядов первого типа $(b)$ находится между кривыми 2 и $3(a)$, соответствующими бифуркации удвоения периода. Область пачечных разрядов второго типа $(c)$ находится между кривыми 4 и $5(a)$, соответствующими бифуркации тора. Кривые 1,6 соответствуют бифуркации Андронова-Хопфа и ограничивают область ритмических колебаний системы (1).

на рис. 1, $a$ цифрами 1 и 6$)$. Эта область лежит в пределах значений проводимости медленных натриевых каналов $g_{N a S}$ от 4 до $12 \mathrm{mS} / \mathrm{cm}^{2}$ и значений стимулирующего тока от 7.8 до $25 \mu \mathrm{A} / \mathrm{cm}^{2}$ (рис. 1,a). За пределами построенной области стационарное состояние системы устойчиво, что соответствует гиперполяризованному состоянию мембранного потенциала (слева от построенной границы) и деполяризованному состоянию (справа от границы).

Пачечные разряды существуют в двух областях, представленных на рис. 1, $a$. Форма пачечных разрядов в этих областях различна. Пачечная активность первого типа в форме дуплетов или мультиплетов с подпороговыми колебаниями в интервалах между пачками наблюдается при значениях параметров $g_{N a S}$ и $I$, лежащих между двумя кривыми бифуркации удвоения периода (штрихпунктирные линии, обозначенные на рис. 1, $a$ цифрами 2 и 3 ).

В диапазоне значений параметров между кривой бифуркации Андронова-Хопфа и бифуркацией удвоения периода ритмическая активность существует в виде синглетных колебаний, при которых один импульс большой амплитуды чередуется с двумя подпороговыми колебаниями малой амплитуды. При увеличении стимулирующего тока бифуркация удвоения периода приводит к появлению пачечной активности в виде дуплетов, в которых происходит чередование двух импульсов большой амплитуды с одним подпороговым колебанием. При дальнейшем увеличении стимула число импульсов в пачке возрастает (рис. $1, b)$.

Пачечная активность второго типа наблюдается в диапазоне значений параметров $g_{N a S}$ и $I$, лежащих между кривыми бифуркации тора (штрихпунктирные линии, обозначенные на рис. 1, $a$ цифрами 4 и 5). Для пачечных разрядов этого типа характерны большое число импульсов в пачке и отсутствие подпороговых колебаний в интервалах между пачками (рис. $1, c$ ).

Между кривыми бифуркации удвоения периода и бифуркации тора в системе существуют только быстрые колебания. Область ритмических колебаний сужается при увеличении $g_{N a S}$ (рис. $\left.1, a\right)$, но области пачечных разрядов обоих типов сохраняются.

\section{2. Механизм возникновения пачечной активности в модели}

Механизм возникновения пачечной активности в модели мембраны ноцицептивного нейрона подробно рассмотрен в нашей предыдущей работе [9]. Рис. 2 иллюстрирует этот механизм для пачечной активности первого и второго типов. Бифуркационная диаграмма быстрой подсистемы на плоскости $(r, E)$, где $r$ служит 

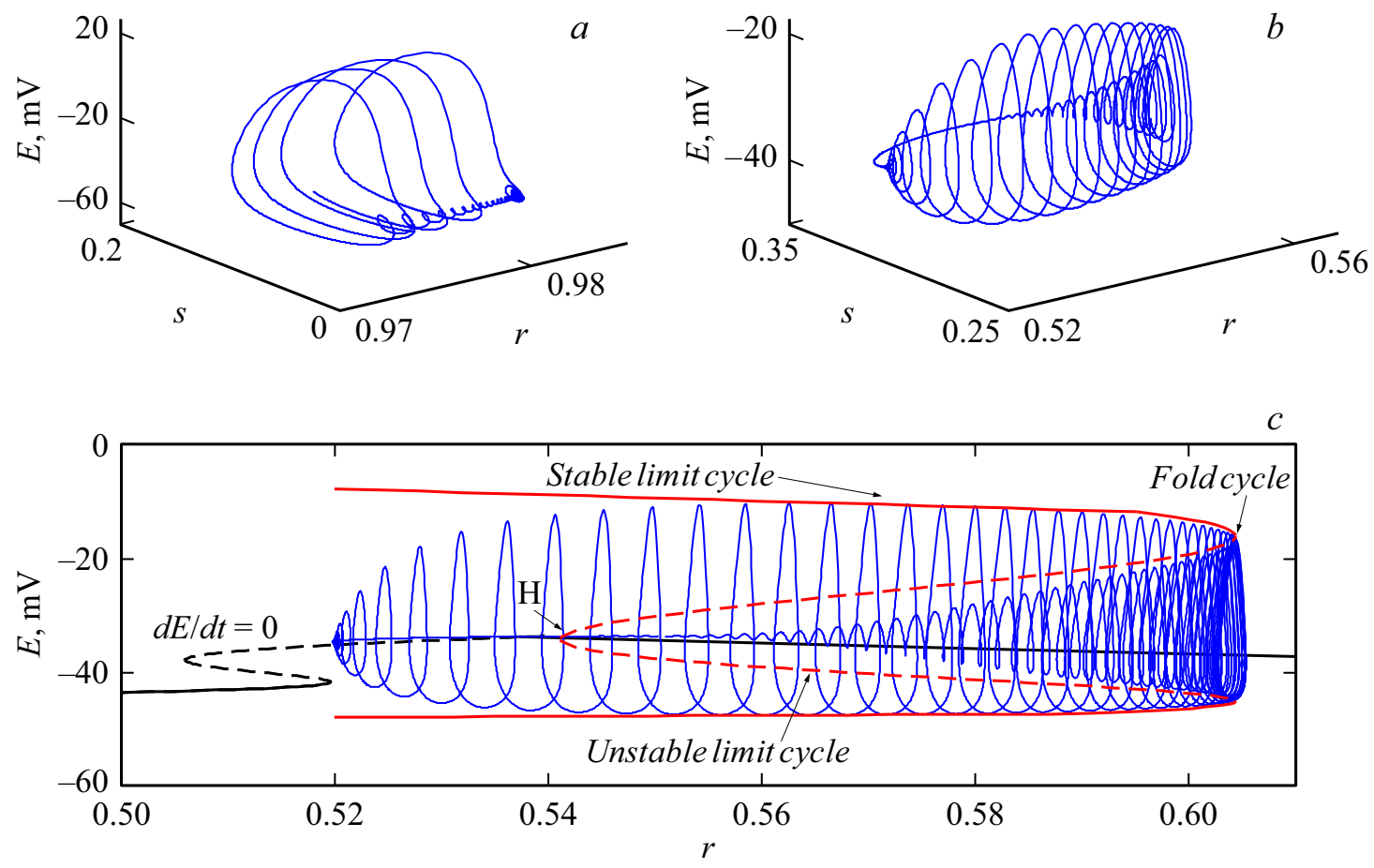

Рис. 2. Траектории системы (1) в пространстве $(r, s, E)$ для пачечной активности первого $(a)$ и второго $(b)$ типов и бифуркационная диаграмма быстрой подсистемы на плоскости $(r, E)$ с наложенной на нее траекторией полной системы $(c)$. Бифуркационная диаграмма содержит ветви стационарных состояний (кривые $d E / d t=0$ ) и устойчивых и неустойчивых периодических орбит (stable limit cycle и unstable limit cycle) и получена при значении $I=23.5 \mu \mathrm{A} / \mathrm{cm}^{2}$.

бифуркационным параметром, включает в себя ветви стационарных состояний (кривые $d E / d t=0)$ (неустойчивых, обозначенных штрихпунктирными линиями, и устойчивых, обозначенных сплошными линиями), и ветви периодических орбит (неустойчивых, обозначенных как unstable limit cycle, и устойчивых - stable limit cycle).

Несмотря на различия в форме пачечных разрядов, механизм их возникновения для пачечной активности первого и второго типов един и связан с прохождением траектории системы через бифуркацию Андронова-Хопфа ( $H$ на рис. 2,c), в которой стационарное состояние системы теряет устойчивость, и система переходит в режим периодических колебаний, а окончание активной фазы пачечной активности связано с прохождением траектории системы через седлоузловую бифуркацию предельного цикла (fold cycle на рис. 2,c). Таким образом, оба типа пачечной активности представляют собой тип пачечного разряда „Хопф/складка“ (subHopf/fold cycle burster) по топологической классификации моделей пачечной активности Ижикевича [19].

\section{3. Переход от ритмической активности к пачечной включает в себя решения типа „утки“ (torus canards)}

Покажем, что переход от быстрых ритмических колебаний к пачечной активности типа subHopf/fold cycle burster при изменении величины стимулирующего тока I включает в себя особые решения типа „утки“, которые в иностранной литературе называются torus canards [11]. Решения типа torus canards представляют собой орбиты, проводящие длительное время вблизи притягивающих (устойчивых) и отталкивающих (неустойчивых) периодических орбит $[11,12]$.

Такие решения возникают во многих моделях нейронов при переходах от режима периодических колебаний к пачечной активности. Решения типа „утки“ обнаружены в модели импульсной активности клеток Пуркинье [12], в модели Хиндмарш-Розе, в модели Морриса-Лекара-Термана и в модели Вилсона-Кована-Ижекевича [13]. Отличительными особенностями этих моделей являются разнообразные бифуркации, ведущие к появлению пачечных разрядов. При этом модель Хиндмарш-Розе является моделью типа subcritical elliptic burster, модель Морриса-Лекара-Термана моделью типа circle/fold-cycle bursting, а модель Вилсона-Кована-Ижекевича - моделью типа fold/fold-cycle bursting [13]. Общим для рассмотренных моделей являются наличие быстрой и медленной динамики, а также наличие седлоузловой бифуркации предельного цикла в структуре бифуркационной диаграммы быстрой подсистемы и бифуркации тора в структуре бифуркационной диаграммы полной системы [13]. Многообразие моделей, в которых встречаются уточные решения, связанные с переходами от режима периодических колебаний к па- 

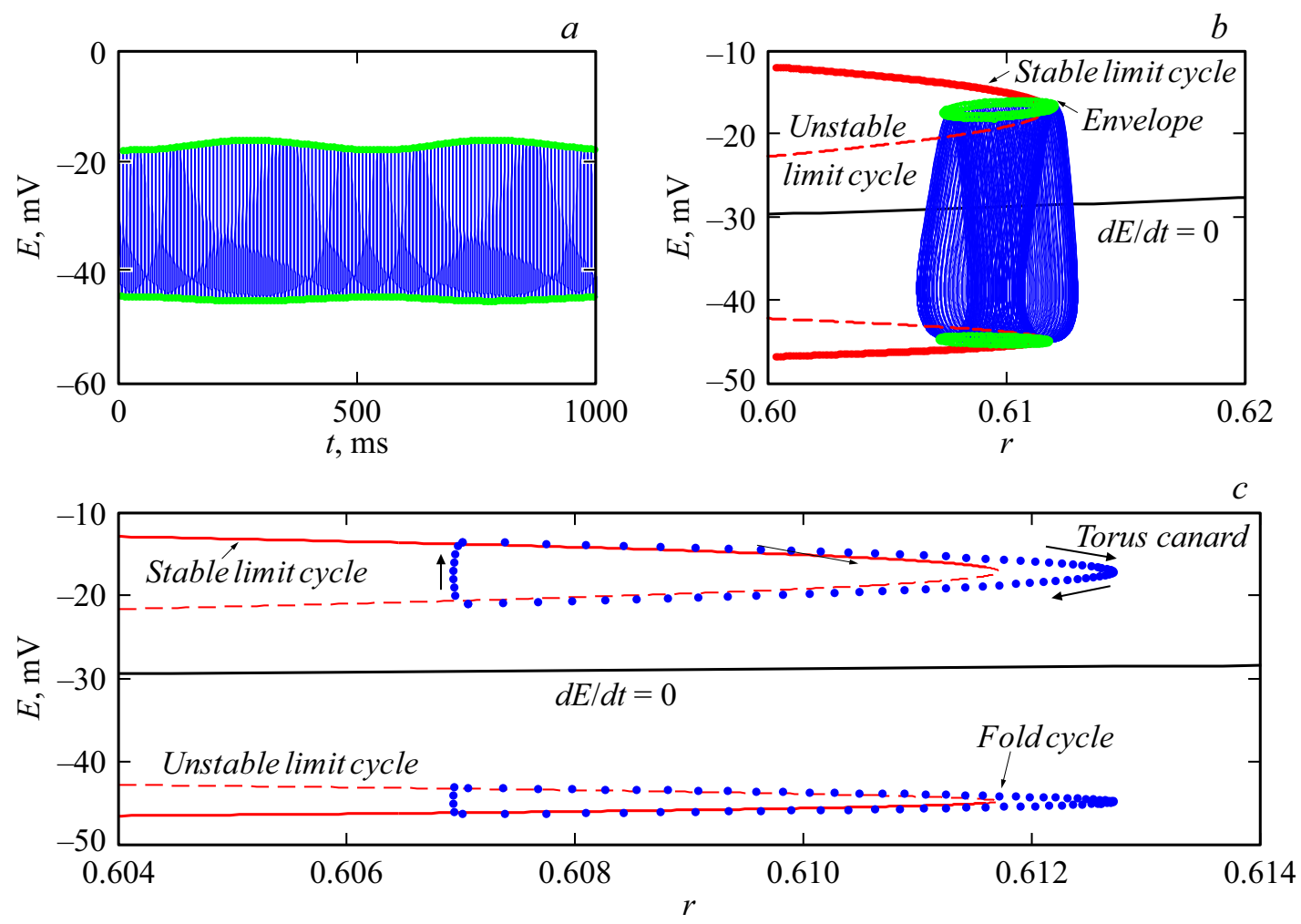

Рис. 3. Колебания типа headless torus canards и бифуркационная диаграмма быстрой подсистемы на плоскости $(r, E)$ при значении $I=22.82 \mu \mathrm{A} / \mathrm{cm}^{2}$. Колебания величины $E$ представляют амплитудно-модулированные колебания с медленно осциллирующей огибающей $(a)$. На бифуркационную диаграмму наложена траектория тора с отмеченной как епvеlope огибающей $(b)$. Траектория headless torus canards вблизи притягивающей (stable limit cycle и отталкивающей (unstable limit cycle) ветвей отмечена штриховой линией (с маркерами в виде кружков) $(c)$.

чечной активности, позволили ряду исследователей [1114] предположить, что решения типа torus canards представляют собой обязательную особенность, характерную для переходов между ритмическими и пачечными разрядами.

В рассматриваемой модели мембраны ноцицептивного нейрона также присутствуют характерные особенности, которые могут указывать на наличие решений типа torus canards. Во-первых, это наличие в структуре бифуркационной диаграммы быстрой подсистемы седлоузловой бифуркации для потери устойчивости предельного цикла. Во-вторых, это потеря устойчивости периодической орбиты полной системы при изменении бифуркационного параметра в точке бифуркации тора, что приводит к появлению пульсирующих волнообразных паттернов с попеременно возрастающей и убывающей амплитудой импульсов, т.е. амплитудно-модулированных колебаний [14].

В целом в рассматриваемой модели при увеличении стимула наблюдается следующая динамика переходов: гиперполяризованное состояние мембраны сменяется периодическими колебаниями, затем пачечными разрядами в виде дуплетов и мультиплетов, ритмической активностью, амплитудно-модулированными колебания- ми, снова пачечным режимом колебаний и, наконец, деполяризованным состоянием мембраны.

Бифуркация тора создает тор, окружающий седлоузловую бифуркацию предельных циклов быстрой подсистемы. Это приводит к слабой амплитудной модуляции периодических орбит. Дальнейшее увеличение стимулирующего тока увеличивает амплитудную модуляцию. В этих условиях траектория полной системы попеременно находится вблизи отталкивающей (неустойчивой) и притягивающей (устойчивой) ветвей периодических орбит быстрой подсистемы.

Увеличение стимулирующего тока вызывает сначала появление траекторий типа „утки без головы“ (headless torus canards), а затем траекторий типа „утки с головой“ (torus canards with heads), и, наконец, переход к пачечной активности типа „Хопф/складка“" (subHopf/fold cycle burster) [13].

В случае траекторий headless torus canards колебания мембранного потенциала напоминают амплитудномодулированные колебания (рис. 3,a), в то время как torus canards with heads напоминают пачечную активность (рис. 4,a). Амплитудно-модулированные колебания состоят из быстрых импульсов и медленно осциллирующей части. Поскольку семейства притягивающих и отталкивающих ветвей периодических орбит встре- 

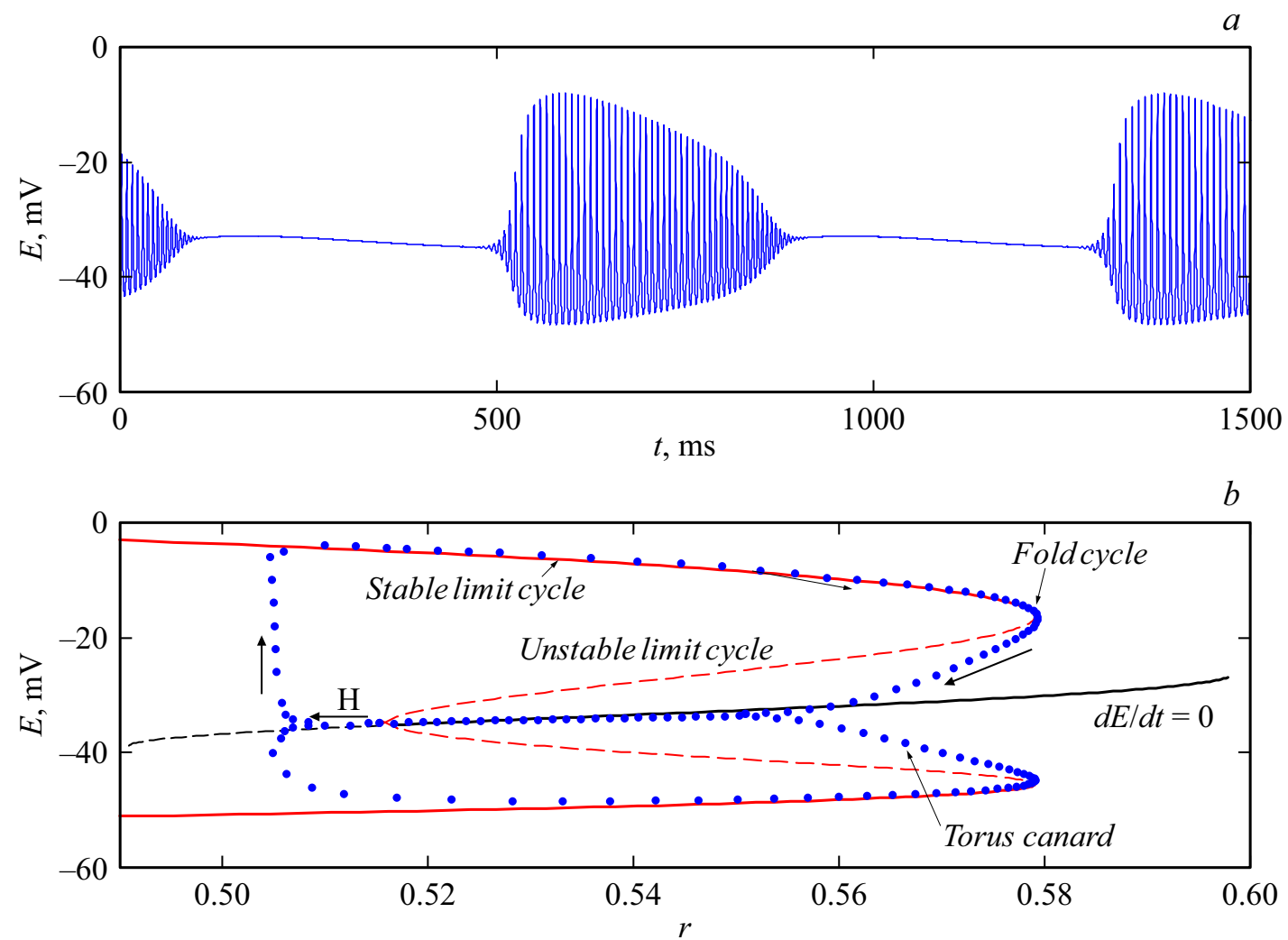

Рис. 4. Колебания типа torus canards with heads и бифуркационная диаграмма быстрой подсистемы на плоскости $(r, E)$ при значении $I=23.12 \mu \mathrm{A} / \mathrm{cm}^{2}$. Колебания величины $E$ представляют собой пачечные колебания $(a)$. Траектория torus canards with heads вблизи притягивающей (stable limit cycle) и отталкивающей (unstable limit cycle) ветвей периодических орбит отмечена штриховой линией (с маркерами в виде кружков) $(b)$.

чаются в седлоузловой бифуркации предельных циклов быстрой подсистемы, обозначенной на рис. 3, $c$ и 4, $b$ как fold cycle, уточные решения torus canards возникают в окрестности именно этой бифуркации.

В случае headless torus canards траектория полной системы проводит некоторое время вблизи внешней (притягивающей) ветви периодических орбит быстрой системы около седлоузловой бифуркации предельных циклов, затем продолжает движение к внутренней (отталкивающей) ветви периодических орбит (рис. 3,b), в результате чего и создается траектория типа „утки без головы“. Эта траектория отмечена на рис. 3,c прерывистой линией (с маркерами в виде кружков) и получена как отображение Пуанкаре на плоскости $(r, E)$ в окрестности седлоузловой бифуркации предельных циклов. Это отображение построено так, чтобы итерации соответствовали локальным экстремумам величины $E$ траектории полной системы.

В случае torus canards with heads траектория полной системы оставляет отталкивающую ветвь периодических орбит и приближается к притягивающей ветви стационарных состояний, затем траектория оставляет ветвь устойчивых стационарных состояний и после некоторой задержки возвращается к ветви устойчивых периодических орбит через бифуркацию Андронова-Хопфа (рис. $4, b$ ). При этом время, в течение которого траектория уточного решения находится вблизи ветви неустойчивых периодических орбит, значительно больше, чем в случае headless torus canards.

\section{Выводы}

Механизмы перехода от ритмической активности к пачечным разрядам в мембране ноцицептивного нейрона включают решения типа torus canards, которые в зависимости от величины стимулирующего тока могут напоминать амплитудно-модулированные колебания или пачечную активность.

\section{Финансирование работы}

Работа выполнена при финансовой поддержке Программы фундаментальных научных исследований государственных академий на 2013-2020 гг. (ГП-14, раздел 64).

\section{Конфликт интересов}

Автор заявляет, что у него нет конфликта интересов. 


\section{Список литературы}

[1] Amir R., Michaelis M., Devor M. // J. Neurosci. 2002. Vol. 22. P. $1187-1198$

[2] Devor M. // Exp. Brain Res. 2009. Vol. 196. P. 115-128.

[3] Kostyuk E.P., Kostyuk P.G., Voitenko N.V. // Neurophysiology. 2001. Vol. 33. P. 303-313.

[4] Goldin E. // J. Exp. Biol. 2000. Vol. 205. P. 575-584.

[5] Waxman S.G., Cummins T.R., Dib-Hajj S.D. et al. // J. Rehabil. Res. Dev. 2000. Vol. 37. P. 517-528.

[6] Kovalsky Y., Amir R., Devor M. // J. Neurophysiol. 2009. Vol. 102. P. $1430-1442$.

[7] Ishikawa K., Tanaka M., Black J. et al. // Muscle Nerve. 1999. Vol. 22. P. 502-507.

[8] Everill B., Kocsis J.D. // J. Neurophysiol. 1999. Vol. 82. P. 700-708.

[9] Дик O.E. // ЖТФ. 2019. Т. 89. С. 465-474.

[10] Дик О.Е., Крылов Б.В., Плахова В.Б. // Биофизика. 2018. T. 63. C. $1141-1145$.

[11] Kramer M.A., Traub R.D., Kopell N.J. // Phys. Rev. Lett. 2008. Vol. 101. P. 0681031-0681034.

[12] Desroches M., Guckenheimer J., Krauskopf B. et al. // Siam Rev. 2012. Vol. 54. P. 211-288.

[13] Burke J., Desroches M., Barry A.M. et al. // J. Math. Neurosci. 2012. Vol. 2. P. 1-30.

[14] Desroches M., Burke J., Kaper T.J. et al. // Phys. Rev. E. 2012. Vol. 85. P. 0219201-0219206.

[15] Elliott A.A., Elliott J.R. // J. Physiol. 1993. Vol. 463. P. 39-56.

[16] Kuznetsov Y.A. Elements of Applied Bifurcation Theory. NY.: Springer, 1995. 593 p.

[17] Rinzel J., Lee Y.S. Nonlinear oscillations in biology and chemistry (Lecture Notes in Biomathematics) / Ed. by H.G. Othmer. NY.: Springer, 1986. 289 p.

[18] Dhooge A., Govaerts W., Kuznetsov Y.A. et al. MatCont and CL_Matcont Continuation toolboxes in MATLAB. Utrecht Univ, 2006. $100 \mathrm{p}$.

[19] Izhikevich E.M. // Int. J. Bifur. Chaos 2000. Vol. 10. P. 11711266. 